\title{
Generalized Hidden Markov Models for Phylogenetic Comparative Datasets
}

$8{ }^{1}$ Department of Biological Sciences, University of Arkansas, Fayetteville, AR, 72701, USA

9 *To whom correspondence should be addressed (jboyko@uark.edu).

10

11 Running head: corHMM: Generalized hidden Markov models

12

13

14

15

16 


\section{Hidden Markov Models for phylogenetic comparative datasets}

\section{Abstract}

25 1. Hidden Markov models (HMM) have emerged as an important tool for understanding the

26 evolution of characters that take on discrete states. Their flexibility and biological sensibility

27 make them appealing for many phylogenetic comparative applications.

2. Previously available packages placed unnecessary limits on the number of observed and hidden states that can be considered when estimating transition rates and inferring ancestral states on a phylogeny.

3. To address these issues, we expanded the capabilities of the R package corHMM to handle $n$ state and $n$-character problems and provide users with a streamlined set of functions to create custom HMMs for any biological question of arbitrary complexity.

4. We show that increasing the number of observed states increases the accuracy of ancestral state reconstruction. We also explore the conditions for when an HMM is most effective, finding that an HMM is an appropriate model when the degree of rate heterogeneity is moderate to high.

5. Finally, we demonstrate the importance of these generalizations by reconstructing the phyllotaxy of the ancestral angiosperm flower. Partially contradicting previous results, we find the most likely state to be a whorled perianth, whorled androecium, whorled gynoecium. The difference between our analysis and previous studies was that our modeling explicitly allowed for the correlated evolution of several flower characters.

44 Keywords: ancestral state reconstruction, discrete character evolution, hidden Markov models, 45 hidden states, stochastic map. 
2 Boyko and Beaulieu

\section{Introduction}

Hidden Markov models (HMMs) are important centerpieces in many biological

49 applications (Eddy, 2004; Yang Lou, 2017). They provide a natural framework for comparative

50 biologists, particularly for relaxing assumptions about homogeneous evolution through time and

51 across taxa without vastly increasing the number of parameters (e.g., Felsenstein \& Churchill,

52 1996; Galtier, 2001; Penny, McComish, Charleston, \& Hendy, 2001; Beaulieu, O’Meara, \&

53 Donoghue, 2013; Beaulieu \& O’Meara, 2016). For instance, simple models of binary character

54 evolution make sense for small, young clades, because a single set of transition rates seems like a

55 reasonable assumption. However, homogeneous rates are unlikely to explain the evolution of the

56 same character across a much larger and older clade in which transition rates may differ

57 dramatically among subclades, perhaps due to correlations with traits that were not included in

58 the model. This observation was the motivation for the development of the hidden rate model

59 (HRM) of Beaulieu et al. (2013), which uses a hidden Markov approach to objectively locate

60 regions of a phylogeny where hidden factors have either promoted or constrained the

61 evolutionary process for a binary character.

62 Within comparative biology, HMMs have been applied as both standalone models

63 (Beaulieu et al., 2013) and in combination with other phylogenetic models (e.g., hidden state-

64 dependent speciation and extinction models, Beaulieu \& O'Meara, 2016). Hidden Markov

65 models can be used to address many problems in comparative biology (Siepel \& Haussler, 2005)

66 and their flexibility allows biologists to create models tailored to their specific hypotheses.

67 However, previous implementations of HMMs for comparative methods have placed limitations

68 on the number of observed and hidden states. For instance, the implementation of the HRM

69 model of Beaulieu et al. (2013) is restricted only to the analysis of binary characters. There is no 


\section{Hidden Markov Models for phylogenetic comparative datasets}

70 mathematical basis for limiting the number of observed states or hidden states in an HMM, and

71 such constraints necessitate a simplification of datasets and candidate models.

72 Here we describe a new version of corHMM that implements $n$-state HMMs. This does

73 not require new algorithms or a different likelihood function. Instead, we optimized and

74 generalized existing code so users can create custom HMMs for any biological question of

75 arbitrary complexity. We have also added a number of "quality of life" improvements that make

76 corHMM much easier to use and interpret, including an implementation of stochastic character

77 mapping (simmap; Bollback, 2006). Additionally, we demonstrate the effectiveness of HMMs to

78 identify rate heterogeneity when it is present, and we outline the informational advantages of

79 increasing the number of observed and hidden states in discrete character data sets. Finally, to

80 demonstrate the importance of this generalization, we apply corHMM to reconstruct the

81 morphology of the ancestral angiosperm flower.

\section{Materials and Methods}

\subsection{Generalizing HMMs}

From a technical standpoint, hidden Markov models have a hierarchical structure that can

86 be broken down into two components: a "state-dependent process" (Fig. 1a,b) and an unobserved

87 "parameter process" (Fig. 1c)(Zucchini, MacDonald, \& Langrock, 2017). In comparative

88 biology, for characters that take on discrete states the standard "state-dependent process" is a

89 continuous-time Markov chain with finite state-space (CTMC-FS). The benefit of a Markov

90 model is its simplicity - to calculate the probabilities of observed discrete states at the tips of a

91 phylogeny all that is required is a tree, a transition model describing transitions among a set of

92 observed states, and frequencies at the root (O’Meara, 2012; Fig. 1a,b). The observed states 


\section{Boyko and Beaulieu}

93 could be any discretized trait such as presence or absence of extrafloral nectaries (Marazzi et al.,

94 2012), woody or herbaceous growth habit (Beaulieu et al., 2013), or diet state across all animals

95 (Román-Palacios, Scholl, \& Wiens, 2019). However, a simple Markov process that assumes

96 homogeneity through time and across taxa is often not adequate to capture the variation of real

97 datasets (e.g. Beaulieu et al., 2013). Under an HMM, observations are generated by a given

98 state-dependent process, which in turn depends on the state of the parameter process. In other

99 words, the observed data are the product of several processes occurring in different parts of a

100 phylogeny and the parameter process is way of linking them. It is initially unknown what the

101 parameter process corresponds to biologically, hence the moniker "hidden" state. Nevertheless,

102 the information for detecting hidden states comes from the differences in how the observed states

103 change. As long as the transitions between observed states of different lineages are more

104 adequately described by several Markov processes rather than a single process, there will be

105 information to detect hidden states (see 3.1 Performance in Simulations).

106 The likelihood of any HMM is obtained by maximizing the standard likelihood formula,

$107 L=P(D \mid \mathbf{Q}, T)$, for observing character states, $D$, across a set of extant taxa, given the

108 continuous-time Markov model $\mathbf{Q}$, and a fixed topology with a set of branch lengths (denoted by

$109 T$ ). For a binary character, $\mathbf{Q}$ is a $2 \times 2$ transition matrix representing the transition rates, whose

110 entries define transitions between the character states, 0 and 1. To form an HMM, we expand $\mathbf{Q}$

111 to accommodate both observed and hidden states. Formally, the HMM can be generalized to

112 include any number of observed states (e.g., 0, 1, 2), and hidden states (e.g., A, B, C). Following

113 Beaulieu and O'Meara (2016), the state space is defined as $o$ being the index of the observed

114 state, $o \in 0,1, \ldots, \alpha$, and $h$ as the index of the hidden state, $h \in A, B, \ldots, \beta$. Thus, a given model

115 will have, in general, $|o| \times|h|$ states. In CorHMM, the model $\mathbf{Q}$ is defined by amalgamating each 


\section{Hidden Markov Models for phylogenetic comparative datasets}

116 of the state-dependent processes and the parameter process specified in the model. For example,

117 if we have state-dependent matrices, $\boldsymbol{R}$,

$$
\boldsymbol{R}_{1}=\left(\begin{array}{ccc}
- & 1 & 2 \\
1 & - & 3 \\
2 & 3 & -
\end{array}\right), \boldsymbol{R}_{2}=\left(\begin{array}{ccc}
- & 5 & 0 \\
4 & - & 7 \\
0 & 6 & -
\end{array}\right)
$$

119 that are related by a parameter-process $\boldsymbol{P}$,

$$
\boldsymbol{P}=\left(\begin{array}{cc}
- & 9 \\
8 & -
\end{array}\right)=\left(\begin{array}{cc}
- & r_{R 1 \rightarrow R 2} \\
r_{R 2 \rightarrow R 1} & -
\end{array}\right)
$$

120 where entries $r_{R 1 \rightarrow R 2}$ and $r_{R 2 \rightarrow R 1}$ define transition rates between the state-dependent processes,

121 we can extend Eq. 2 of Tarasov (2019) to amalgamate these processes,

$$
\mathbf{Q}=A\left(\boldsymbol{R}_{1}, \boldsymbol{R}_{2}, \boldsymbol{P}\right)=\left(\begin{array}{cccccc}
- & 1 & 2 & 9 & 0 & 0 \\
1 & - & 3 & 0 & 9 & 0 \\
2 & 3 & - & 0 & 0 & 9 \\
8 & 0 & 0 & - & 5 & 0 \\
0 & 8 & 0 & 4 & - & 7 \\
0 & 0 & 8 & 0 & 6 & -
\end{array}\right)
$$

122 This matrix can be understood as a block matrix where the diagonal blocks are the state-

123 dependent processes $\boldsymbol{R}_{1}$ and $\boldsymbol{R}_{2}$, and the off-diagonal blocks are the parameter process, $\boldsymbol{P}$, that

124 describe transitions between $\boldsymbol{R}_{\mathbf{1}}$ and $\boldsymbol{R}_{2}$. It is important to note that although we amalgamate the

125 matrices above, it is still possible for users to modify the transitions between hidden states.

127 evolution of multiple characters (Pagel, 1994). For example, consider a case of two binary 128 characters where trait 1 defines the presence or absence of fleshiness of fruits, and trait 2 defines

129 whether or not the fruits are animal-dispersed. At most there are four binary combinations of 130 these characters (i.e., 00, 01, 10, and 11). But, it can also be coded as a single multistate 131 character, where $1=$ dry fruits not dispersed by animals, $2=$ dry fruits dispersed by animals, 1323 =fleshy fruits not dispersed by animals, and 4=fleshy fruits dispersed by animals. Therefore, 133 transforming binary combinations to multistate characters also applies for two characters with a 


\section{Boyko and Beaulieu}

134 different number of observed states. In other words, one character could be binary (e.g., dry vs.

135 fleshy fruit) and the other could be multistate (e.g., fruits dispersed mechanically, by wind, or by 136 animal).

\subsection{Simulation Study}

We conducted a set of simulations to address two broad goals. First we tested whether

140 there is an informational advantage to increasing the number of observed states by comparing

141 two-state, three-state, and four-state datasets. Our second goal was to test the ability of hidden

142 Markov models to detect varying degrees of rate heterogeneity. We then linked these goals

143 together by testing whether HMMs can recover some of the informational content of unobserved

144 characters through the use of hidden states. These simulations are in no way exhaustive, but

145 represent a set of reasonable questions that many beginning users might have about the behavior 146 of HMMs.

\subsubsection{Increasing the number of observed characters or states}

149 To test the behavior of two-state, three-state, and four-state datasets we relied on 150 ancestral state reconstruction (ASR) at nodes. ASR is a widely-utilized feature of corHMM, and

151 it is important to know the accuracy of multistate ancestral reconstructions. Additionally, using

152 ancestral states gives us a direct means to compare models with different datasets. A 250-tip

153 phylogeny was simulated (birth rate set to 1 event $\mathrm{Myr}^{-1}$, and death rate of 0.5 events $\mathrm{Myr}^{-1}$ ) to

154 be used as a fixed tree with a root age of 12.46 Myr and mean branch length of 0.89 Myr.

155 Datasets were simulated using transition rates sampled from a truncated normal distribution $(\mu=$

$1561, \sigma=0.5)$, which were then scaled to have mean rates of $0.1,1.0$, or 10 transitions $\mathrm{Myr}^{-1} \mathrm{by}$ 


\section{Hidden Markov Models for phylogenetic comparative datasets}

157 dividing the rate matrix by the sum of the diagonal and then multiplying by the desired scalar.

158 This resulted in a range of evolutionary rates where the expected number of transitions ranged

159 from $\sim 21,210$, or 2100 transitions across the entire tree depending on the mean rate. For each

160 transition model, 100 datasets were simulated. The transition rates of each dataset were then

161 estimated and their maximum likelihood estimates were used to infer marginal probabilities of

162 each character state across the tree. This procedure was repeated 10 times.

163 An underappreciated concern with evaluating models that differ in the number of

164 observed states is that the probability of guessing the correct state without any additional

165 information is simply $1 / k$ states. This could, in theory, inflate the accuracy of datasets with fewer

166 states even though the tip states themselves provide no information about the ancestral states

167 when the rates are high (Schultz, Cocroft, \& Churchill, 1996; Sober \& Steel, 2011, 2014). To

168 deal with this issue, we also calculated the mutual information, measured in bits, about ancestral

169 states from each dataset and model (Cover \& Thomas, 1991; Sober \& Steel, 2011). Specifically,

170 mutual information is a measure of how much ancestral state uncertainty is reduced by knowing

171 the tip states (details of our derivation are given in S1). The initial uncertainty, or unconditional

172 entropy, is set by the model - given a model of evolution and no knowledge of the extant tips,

173 how uncertain is the best guess of the ancestral states? The remaining uncertainty after ASR, or

174 conditional entropy, is given by the combination of the model and the tip states - given the

175 model of evolution and knowledge of the extant tips, how uncertain is the best guess of the

176 ancestral states? It is important to note that information, just like ancestral state reconstruction, is

177 highly correlated with the model of evolution, and thus any results related to information will

178 take on the assumptions of the model. 
8 Boyko and Beaulieu

We define information as the difference between the unconditional entropy of the node states, $H\left(X_{v}\right)$, and the entropy of the node states conditioned on the data, $H\left(X_{v} \mid X_{h}=D\right)($ Cover

$181 \&$ Thomas, 1991). The unconditional entropy of node $v$ is defined as:

$$
H\left(X_{v}\right)=-\sum_{i=1}^{k} \pi\left[X_{v}=i\right] \log _{2}\left(\pi\left[X_{v}=i\right]\right)
$$

182 where $\pi\left[X_{v}=i\right]$ is the prior probability of a node taking a particular state. For the root, the prior 183 depends on user choice, as there are several options (Yang, Kumar, \& Nei, 1995; Pagel, 1999;

184 FitzJohn, Maddison, \& Otto, 2009). Here we assume the prior probability on the root node is the 185 expected equilibrium frequency, $\pi$, which is calculated directly from the transition model by 186 solving $\pi \boldsymbol{Q}=0$. This aligns our expectation of the root node with all other internal nodes such 187 that, in the absence of information from the tips, the probability of a particular state is assumed to be drawn from the equilibrium frequencies. In other words, the information of the tip states decreases as rates increase and, ultimately, the probability of a node state becomes completely determined by the model. We define the conditional entropy as:

$$
H\left(X_{v} \mid X_{h}=D\right)=-\sum_{i=1}^{k} P\left[X_{v}=i \mid X_{h}=D\right] \log _{2}\left(P\left[X_{v}=i \mid X_{h}=D\right]\right)
$$

191 where $P\left[X_{v}=i \mid X_{h}=D\right]$ is the conditional probability that a node is fixed as being in state $i$ 192 given the probability of observing the tip data (which is just the marginal probability of state $i$ ).

193 In particular, we are interested in the average entropy of a node for all states $i \ldots k$, given we 194 observe a particular dataset, $X_{h}=D$. Thus, the conditional entropy will vary by node, but the 195 unconditional entropy is set by the model. To produce a measure of mutual information between 196 the observations at the tips and estimates at internal nodes, we take the difference between the 197 conditional entropy and the unconditional entropy and average across all nodes. However, the 


\section{Hidden Markov Models for phylogenetic comparative datasets}

198 unconditional entropies will be greater for datasets that include more states because

199 unconditional entropy sets the upper limit of what is possible to learn. This alone could

200 contribute to large informational differences between models with different numbers of observed

201 states. Therefore, we also measure the proportion of maximum information gained

$\left(\frac{\text { mutual information }}{\text { unconditional entropy }} \times 100 \%\right)$.

203

204

205

206

207

208

209

210

211

212

213

214

215

216

217

218

219

220

\subsubsection{Evaluating hidden Markov models}

We evaluated the ability to detect rate heterogeneity by simulating data under an HMM. As outlined above (see 2.1 Generalizing $H M M s$ ), there are two major axes along which an HMM differs from standard Markov models. First, we varied the magnitude of the difference in the state-dependent process by simulating data under a model where there was: (1) no difference between the state-dependent processes $\left(\boldsymbol{R}_{\mathbf{1}}=\boldsymbol{R}_{2}\right)$, (2) a 2-fold difference in rates between the state-dependent processes (e.g. if $\boldsymbol{R}_{1}$ 's mean rate was $1 \mathrm{Myr}^{-1}, \boldsymbol{R}_{2}$ mean rate would be $2 \mathrm{Myr}^{-1}$ ),

(3) a 10-fold difference between the state-dependent processes, and (4) a covarion-like trait model in which within $\boldsymbol{R}_{\mathbf{1}}$ all transitions occur freely, but for $\boldsymbol{R}_{2}$ all transition rates are zero, and evolution is essentially "turned off" (Penny et al., 2001). For all simulation scenarios, we set the parameter-process to have equal transition rates between state-dependent processes. In addition to examining ancestral state reconstruction at nodes, we also used the new makesimmap to assess how well the model captures the expected number of character changes within and among all branches in the tree. For each of the 150 datasets simulated above, we evaluated 100 simmaps per model by counting the number of transitions for a given simmap.

Next, we tested the impact of the magnitude of the asymmetry in the underlying parameter-process. We simulated data where the state-dependent process always differed by 2fold, but for the underlying parameter-process there was: (1) no difference in transition rate 
10 Boyko and Beaulieu

$221\left(r_{R 1 \rightarrow R 2}=r_{R 2 \rightarrow R 1}\right),(2) 1.5 \times$ faster transition rate to the slower rate class $\left(r_{R 1 \rightarrow R 2}>r_{R 2 \rightarrow R 1}\right),(3) 2 \times$

222 faster transition rate to the slower rate class, (4) 10× faster transition rate to the slower rate class.

223 For each of the models described, we used simulated 500-tip phylogeny with a root age of 15.43

224 Myr (birth rate set to 1 event $\mathrm{Myr}^{-1}$, and death rate of 0.7 events $\mathrm{Myr}^{-1}$ ).

225 Finally, we examined how much information is available when we allow for hidden states

226 to be observed at the tips. We used the same data generated from simulations examining state-

227 dependent differences, but this time we did not remove the hidden state. We then fit a Markov

228 model to this full dataset and compared it to models in which the "second character" remained

229 unobserved.

2312.3 Case study: reconstructing the ancestral angiosperm flower

\section{2.3.1 Background}

233 Understanding the origin of flowering plants is widely considered to be one of the most

234 important goals of systematic botany. In a recent study, (Sauquet et al., 2017) compiled an

235 extensive database of floral characteristics and attempted to reconstruct the morphology of the

236 ancestral angiosperm flower. Sauquet et al. (2017) did not present a single answer for the

237 ancestral state because there were several possible state combinations depending on the method

238 used and uncertainty associated with each of those estimates. Nonetheless, their hypothetical

239 diagram of the ancestral flower as having a whorled perianth, whorled androecium, and spiral

240 gynoecium proved controversial. For example, Sokoloff, Remizowa, Bateman, \& Rudall (2018)

241 disputed this depiction of the ancestral phyllotaxy, suggesting that some of the characters were

242 scored incorrectly and that it seemed improbable that state combinations that are rare in the data

243 could be the ancestral state. Sokoloff et al. (2018) instead prefer the hypothesis that the ancestral 


\section{Hidden Markov Models for phylogenetic comparative datasets}

244 flower was either entirely whorled or entirely spiraled. In response, Sauquet et al. (2018)

245 rescored the disputed characters and reanalyzed the dataset using the same methods as the

246 original study. Their Bayesian analyses conformed to the predictions of Sokoloff et al. (2018),

247 but remained highly uncertain overall. A limitation of the original study was the fact that "no

248 comparative method exists yet to account for the potential correlation of more than two discrete

249 characters" (Sauquet et al., 2017, but see Beaulieu \& Donoghue, 2013). Given that flowers are

250 highly integrated structures with potentially several developmental constraints the correlation

251 between states seems a necessary prerequisite to study their evolution (Sauquet et al., 2017,

252 2018; Sokoloff et al., 2018). Treating the phyllotaxy of the perianth, androecium, and

253 gynoecium as independent represents a major assumption with potentially large consequences on

254 the ancestral state reconstruction. Indeed, Sauquet et al. (2017) conducted several pairwise

255 correlation analyses and found that the phyllotactic patterns of these focal characters were

256 strongly correlated.

\subsubsection{Worked example and methods}

259 We limited ourselves to including only the characters related to the phyllotaxy of the

260 perianth, androecium, and gynoecium. Although it is possible to include other characters, given

261 the corresponding increase of parameter space, we suspect that we would not have the power to

262 accurately infer the model and ancestral states (O'Meara et al., 2016). The dataset of Sauquet et

263 al. (2018) has several polymorphic species as well as species for which some of the tip states are

264 unknown. Therefore, we analyzed three separate datasets: $(1)$ no uncertain taxa $(\mathrm{n}=295),(2)$

265 polymorphic species included $(n=297)$, and (3) all taxa included $(n=780)$. We treated the

266 phyllotaxy of the perianth, androecium, and gynoecium as either "whorled" or "spiral" and 


\section{Boyko and Beaulieu}

267 polymorphic species are coded to have both states. The choice dataset has major implications for

268 model performance because corHMM will exclude state combinations that are absent from the

269 dataset. However, the inclusion of either polymorphic or unknown states for taxa will expand

270 state space and thus increase the number of parameters that need to be estimated. Finally, we use

271 the C series phylogeny of Sauquet et al. (2017) in which Amborella is constrained as the sister to

272 all remaining angiosperms and Monocotyledoneae, Ceratophyllaceae, and Eudicotyledoneae are

273 constrained to form a monophyletic group.

274 In our case, we have three data columns each with two observed states. Because this

275 dataset contains two or more columns of trait information, each column is automatically

276 interpreted as an evolving character. In these cases, corHMM will also automatically remove dual

277 transitions from the model since that would constitute two or more evolutionary events (Pagel,

278 1994; Maddison, Midford, Otto, \& Oakley, 2007). However, previous work has suggested that

279 dual transitions are possible and likely in this system (Sauquet et al. 2018; Sokoloff et al. 2018).

280 Thus, we include both models in which dual transitions are allowed and disallowed. Our analysis

281 without hidden states include three different model structures: model="ER" (equal rates),

282 model="SYM" (symmetric rates), and model="ARD" (all rates differ). The other options

283 used (rate. cat=1 and nstarts=10) specify that no hidden states are to be used and that the

284 maximum likelihood search will be performed 10 additional times with different initial

285 parameters.

286 We also include a set of analyses in which hidden states are present because it is likely that

287 there are unobserved characters which influence the evolution of the angiosperm flower. We 288 include four hidden state models: ER/ER, SYM/SYM, ARD/ARD, and ER/ARD. Each of these 289 models allows for the possibility of rate heterogeneity through the inclusion of a hidden state, 


\section{Hidden Markov Models for phylogenetic comparative datasets}

290 however the state-dependent processes differ. In the $E R / E R$ model all changes between states

291 occur at the same rate within a state-dependent process, but the magnitude of change can depend

292 on the underlying parameter process. The $S Y M / S Y M$ model specifies that within character

293 changes are equally probable, but some characters may change faster than others. The ARD/ARD

294 model specifies that there could be a bias towards a particular state, but this state may differ

295 depending on whether the lineage is in $\boldsymbol{R}_{\mathbf{1}}$ or $\boldsymbol{R}_{2}$. Finally, ER/ARD is a hybrid model which

296 includes aspects of the equal rates model and all rates differ model.

\section{3. Results}

2983.1 - Performance in Simulation

\section{1 .1 - Increasing the number of observed characters or states}

300 Overall, the accuracy of an ancestral state reconstruction is predominately a function of

301 the transition rates, but there are regions of parameter space where the number of states is

302 influential (Fig. 2a). For example, all datasets generally inferred the correct ancestral state at low

303 rates and datasets with more states performed better at intermediate rates. However, when

304 viewed in terms of information, datasets that contained just two states showed detectable

305 informational loss when compared to the three- and four-state datasets. In fact, across all

306 scenarios - low, intermediate, and especially at the highest rates — datasets with more states

307 consistently showed more informational gain relative to the maximum information content for a

308 given number of states (Fig. 2b). We suspect this largely reflects the impacts of homoplasy when

309 the number of character states are restricted in the model (Sanderson \& Donoghue, 1989; Steel \&

310 Penny, 2005). This is not to say that more character states are always necessary for accurate

311 ASR. Rather, we demonstrate that there are cases when additional characters or character states 


\section{Boyko and Beaulieu}

312 enhance the accuracy of an ancestral state reconstruction and those datasets have a signal of

313 increased information.

\subsection{2 - Evaluating hidden Markov models}

317 at nodes, appears largely unaffected by the inclusion of hidden states regardless of differences in

318 the state-dependent processes (Fig. 3a). However, the amount of information gained depends on

319 both the use of an HMM and the presence of strong differences between the state-dependent

320 processes (Fig. 3b). These seemingly contradictory results are a consequence of how we

321 calculate information. Model uncertainty certainly comes from the increase in parameters of

322 HMMs relative to standard Mk models and manifests in both increased model complexity and an

323 increased number of potential ancestral states. The increase in possible ancestral states results in

324 a higher unconditional entropy, which can actually lead to greater informational content even

325 when the ancestral states are not known with as much certainty as a Mk model. However, as we

326 show in section 3.1.1 increasing the number of observed states improved ancestral state

327 reconstruction accuracy, despite a greater number of estimated parameters, and so this does not

328 solely account for the greater ancestral state accuracy of a Mk over an HMM. We suspect it is

329 also due to datasets fit under an HMM have added uncertainty applied to the tips because it is

330 initially unknown which hidden state a particular taxon occupies. The greater uncertainty at the

331 tips is likely the reason why we observe Mk models outperforming HMMs in ancestral state

332 reconstruction, and the greater uncertainty of the model is likely why HMMs are able to extract

333 more information from a given dataset. 


\section{Hidden Markov Models for phylogenetic comparative datasets}

We found that when the generating model does not have state-dependent differences, the

335 HMM does not pickup significant rate variation and resembles the character history implied by

336 the standard Markov model (Fig. 4a-c). When there was no difference between the state-

337 dependent processes, $2.6 \%$ of datasets had an AICc difference greater than 2 in support of an

338 HMM over a Markov model. Whereas $100 \%$ of datasets supported an HMM when data was

339 simulated under a covarion-like model. These findings suggest that HMMs are supported in

340 datasets where rate heterogeneity is present and this can be seen qualitatively through simmap

341 reconstructions (Fig. 4d-f). We found little effect of altering the transition rate bias of the

342 parameter process on either ancestral state reconstruction (Fig. S1a) or information content (Fig.

343 S1b).

344 Unsurprisingly, observing the "second character" states increased the amount of

345 information (Fig. 5). However, as the state-dependent processes became more distinguishable,

346 the informational gap between an HMM and including the observed second character decreased.

347 In other words, when the evolution of an observed character changes across the phylogeny, an

348 HMM is able to extract additional information from a dataset.

Across all three datasets our best supported model was $E R / E R$, a two rate class model

352 with both state-dependent processes being equal rates (Table 1; Table S1). Because our

353 modeling set included a wide range of complexity ranging from 1 estimated rate (ER) to 114

354 estimated rates (ARD/ARD), we used AIC weights to calculate the model averaged ancestral

355 states for datasets individually. For all three datasets we find that an entirely whorled angiosperm

356 flower is the most likely state (Table S2). However, we found that the preferred ancestral state is 


\section{Boyko and Beaulieu}

357 highly variable and dependent on the model and the entirely whorled angiosperm flower is likely

358 a reflection of the ER/ER model's high AIC weight within the set of tested models (Table 1;

359 Table S1). For several of the models we found that the parameter estimates reached the upper

360 limit of the transition rates allowed (Table S3). This could be reflection of a lack of adequate

361 data, too many unknown and polymorphic state combinations, and/or unrealistic models included

362 in the set. However, none of the transition rates estimated reached the upper limit for any of the

363 best supported models.

\section{Discussion}

Hidden Markov models are an essential tool for inferring character states across

367 phylogenies. The new version of corHMM, expands the array of potential uses of HMMs by

368 increasing the number of possible character states and allowing users to construct custom

369 models. In addition, we demonstrated the informational advantages of using hidden Markov

370 models versus simple Markov models. Users interested in hypothesis-driven model construction

371 are encouraged to read through the vignette associated with the corHMM package. This vignette

372 fully describes how to use the package and includes several examples of how to take a biological

373 hypothesis and codify it into an explicit HMM.

374 Information theory has mainly been discussed in a theoretical context and rarely used in

375 practice to understand empirical trait evolution (Mossel, 2003; Mossel \& Peres, 2003; Townsend

376 \& Naylor, 2007; Sober \& Steel, 2011, 2014; Gascuel \& Steel, 2014). In this paper, we have

377 introduced a measure for the amount of information that the tips provide the nodes during

378 ancestral state reconstruction. Two important caveats of this measure of information. First, the

379 data and model are taken as fixed. These are not uncommon assumptions in phylogenetic 


\section{Hidden Markov Models for phylogenetic comparative datasets}

comparative methods. For example, if one is to interpret an ancestral state reconstruction it comes with the implicit assumption that the model accurately describes the evolution of the traits

382 (Beaulieu \& O’Meara, 2019). Second, mutual information, as we have defined it, only provides

383 information relative to the specified model and specified tips. A model which is more uncertain

384 about any ancestral state, such as an equal rates model, is likely to have a more informative

385 ancestral state reconstruction because any deviation from an uninformed ancestral state is due to

386 the particular values of the tip states. This does not make the equal rates model better than

387 alternatives nor do we advocate for the use of information to assist in model selection. Instead,

388 mutual information provides insight into the interaction between the model and tip states. Higher

389 information of particular nodes could be indicative of an area of the phylogeny where the

390 model's equilibrium frequencies were different from the ancestral state reconstruction and thus

391 the tips provided the major explanation of the ancestral state. Mutual information is also highly

392 correlated with the rates of evolution and has the intuitive property that as rates of evolution (or

393 time) increase the information that the tips provide to the nodes decreases (Sober \& Steel, 2011).

394 It is important to have a biologically realistic model of trait evolution when conducting an

395 ancestral state reconstruction. With the generalizations made to corHMM we have provided two

396 distinct ways to increase the realism of phylogenetic comparative modeling. First, we have

397 allowed for the correlated evolution of several characters and states. Whether traits are correlated

398 because of underlying pleiotropy leading to genetic correlation (Conner et al., 2011) or selective

399 covariance (Mahler, Revell, Glor, \& Losos, 2010), at the macroevolutionary scale they are better

400 understood in a holistic context rather than independently evolving subunits. Second, the

401 inclusion of hidden states allows for more detailed descriptions of the evolutionary process.

402 State-dependent processes can differ in both rate and structure and thus provide a description of 


\section{Boyko and Beaulieu}

403 heterogeneity in the tempo and mode of evolution. However, these generalizations do not exist

404 without cost. Increased complexity of models leads to greater parameterization which can lead to

405 poor model performance (Grundler \& Rabosky, 2020). Thus, as others recommend, we suggest

406 having multiple working hypotheses (Chamberlin, 1890; Platt, 1964; Mayr, 1997; Burnham \&

407 Anderson, 2002). The generalizations and tools available in corHMM allow for the construction

408 of a carefully defined set of candidate models which can be compared in an information theoretic

409 context. The Akaike Information Criterion (AIC) applies the principles of parsimony and

410 represents a trade-off between bias and variance as a function of the dimension of the model

411 (Forster \& Sober, 1994). Combining AIC with a carefully constructed set of models leads to

412 multi-model inference. Rather than focusing on a single best model, we can focus on the

413 parameters from the set of our best supported models (Burnham \& Anderson, 2002; Caetano,

414 O’Meara, \& Beaulieu, 2018). It is, therefore, just as important to include Mk models alongside

415 HMMs because in cases where the increased parameterization of an HMM are unnecessary,

416 alternative models with less parameterization are available as simpler explanations.

417 We have demonstrated that there is a potential informational and accuracy advantage of

418 including additional states and characters in a simulation setting (3.1.1 Increasing the number of

419 observed characters or states). However, it remained to be seen whether modeling the correlated

420 evolution of multiple characters would impact the ancestral state in an empirical example and

421 whether those results match biological expectations. The controversy surrounding the phyllotaxy

422 of the ancestral angiosperm flower is a particularly appropriate case study for the generalized

423 version of corHMM, as it not only allows for the dependent evolution of several discrete

424 characters but also includes hidden states as a fitting addition to help describe the heterogeneous

425 evolution of angiosperms. We presented three different datasets, each allowing for a different 


\section{Hidden Markov Models for phylogenetic comparative datasets}

426 level of polymorphism, uncertainty, and number of tip states. In our first dataset, we excluded all

427 polymorphic species and any species with an unknown tip state. In this case, we found a two rate

428 class $E R / E R$ model was favored and the most likely ancestral state of the floral phyllotaxy was

429 entirely whorled. This is in contrast to previous work which used a similarly constrained dataset

430 and suggested either an ambiguous state (Maximum Parsimony result), an entirely spiral floral

431 phyllotaxy (Maximum Likelihood result), or a spiral perianth, whorled androecium, and spiral

432 gynoecium (reversable jump Monte Carlo Markov Chain result) (Sauquet et al. 2018 - Appendix

433 S2, V7Csub1).

434 Previous work posed the question of whether we should dismiss ancestral state

435 combinations not observed among living species (Sauquet et al. 2018). The first dataset we

436 presented excluded any trait combinations not observed in the data. However, the other two

437 datasets allow for an ancestral state combination that was never directly observed at the tips

438 because these analyses include tips where the states are not completely known. In both cases, we

439 found the hidden rates model $E R / E R$ where dual transitions are allowed to be the best supported

440 and the model averaged most likely ancestral state was an entirely whorled floral phyllotaxy.

441 Thus, across all datasets we found that the ancestral state combination was one of the most

442 common tip state combinations. However, this does not mean a combination of states unknown

443 in any extant species is impossible. In fact, we find that the preferred ancestral state is highly

444 variable and dependent on the model (Table 1; Table S1) and the entirely whorled angiosperm

445 flower is likely a reflection of the ER/ER model's high AIC weight within the set of tested

446 models. This means that should a more realistic model be introduced in the set we could find a

447 very different answer and highlights the importance of having a set of biologically realistic 448 models. 
20 Boyko and Beaulieu

\section{Conclusion}

Although there is a growing consensus that phylogenies and their associated methods are

451 being used in ways that exceed what they can infer (Losos 2011; Maddison and FitzJohn 2015;

452 Rabosky and Goldberg 2015; Cooper et al. 2016), we have shown that there is still under-utilized

453 information in phylogenetic comparative datasets. First, HMMs extract signals of rate

454 heterogeneity when it is present and, equally important, do not falsely locate signals where they

455 are absent. Second, increased trait depth adds new information and consistently improves

456 ancestral state reconstruction estimates. Indeed, as datasets continue to grow, so will the

457 analytical power that biologists have for testing complex models of evolution. Finally, the

458 inclusion of correlated trait evolution and hidden states is relevant beyond theoretical

459 considerations and we have shown that these generalizations can change the results of an

460 ancestral state reconstruction in empirical datasets. There is still a great deal of uncertainty in the

461 reconstruction of the ancestral phyllotaxy of angiosperms, but by using AIC weighted marginal

462 probabilities we have been able to take into account different biological explanations of floral

463 evolution, eventually finding support an entirely whorled perianth, androecium, and gynoecium.

464 Although hidden Markov models are not a perfect substitute for real observation of a hidden

465 character, they make for a tractable and a biologically reasonable description of heterogeneity in

466 the evolutionary process over long time scales.

468 Availability

469 This open source software is written entirely in the R language and is freely available through the

470 Comprehensive R Archive Network (CRAN) at:

471 https://cran.r-project.org/web/packages/corHMM/index.html. 
21 Hidden Markov Models for phylogenetic comparative datasets

472 The scripts used, simulation results, model fits, and case study data are available at:

473 https://datadryad.org/stash/dataset/doi:10.5061/dryad.vx0k6djpg.

475 Acknowledgements

476 We thank Hervé Sauquet and an anonymous reviewer for their insightful comments on an earlier

477 version of this paper. We also thank members of the Beaulieu lab and colleagues at the 478 University of Arkansas for their comments and for general discussions of the ideas presented 479 here. Finally, we would like to specifically thank Andrew Alverson, Brian O'Meara, and Adam 480 Siepielski for their insightful critiques and helpful edits on an earlier version of this manuscript.

\section{Conflict of Interest}

483 None declared.

485 Author Contributions

486 J.D.B. and J.M.B. designed research; J.D.B performed research and analyzed data; and J.D.B. 487 and J.M.B. wrote the paper.

489 References

490 Beaulieu, J. M., \& Donoghue, M. J. (2013). Fruit Evolution and Diversification in Campanulid 491 Angiosperms. Evolution, 67(11), 3132-3144. doi:10.1111/evo.12180

492 Beaulieu, J. M., \& O’Meara, B. C. (2016). Detecting Hidden Diversification Shifts in Models of 493 Trait-Dependent Speciation and Extinction. Systematic Biology, 65(4), 583-601. 494 doi:10.1093/sysbio/syw022 
22 Boyko and Beaulieu

495 Beaulieu, J. M., \& O’Meara, B. C. (2019). Diversity and skepticism are vital for comparative biology: a response to Donoghue and Edwards (2019). American Journal of Botany, 106(5), 613-617. doi:10.1002/ajb2.1278

Beaulieu, J. M., O’Meara, B. C., \& Donoghue, M. J. (2013). Identifying Hidden Rate Changes in the Evolution of a Binary Morphological Character: The Evolution of Plant Habit in Campanulid Angiosperms. Systematic Biology, 62(5), 725-737.

Bollback, J. P. (2006). SIMMAP: stochastic character mapping of discrete traits on phylogenies. BMC Bioinformatics, 7(1), 88.

Burnham, K. P., \& Anderson, D. R. (2002). Model Selection and Multimodel Inference: A Practical Information-Theoretic Approach (2nd ed.). New York: Springer-Verlag. Retrieved from https://www.springer.com/gp/book/9780387953649

Caetano, D. S., O’Meara, B. C., \& Beaulieu, J. M. (2018). Hidden state models improve statedependent diversification approaches, including biogeographical models: HMM AND

511 Chamberlin, T. C. (1890). The method of multiple working hypotheses. Science, 15(366), 92-96.

512 Conner, J. K., Karoly, K., Stewart, C., Koelling, V. A., Sahli, H. F., \& Shaw, F. H. (2011). Rapid Independent Trait Evolution despite a Strong Pleiotropic Genetic Correlation. The

515 Cooper, N., Thomas, G. H., \& FitzJohn, R. G. (2016). Shedding light on the 'dark side' of phylogenetic comparative methods. Methods in Ecology and Evolution, 7(6), 693-699.

518 Cover, T. M., \& Thomas, J. A. (1991). Elements of information theory. New York: Wiley. 519 Eddy, S. R. (2004). What is a hidden Markov model? Nature Biotechnology, 22(10), 1315-1316. doi:10.1038/nbt1004-1315 
23 Hidden Markov Models for phylogenetic comparative datasets

521 Felsenstein, J., \& Churchill, G. A. (1996). A Hidden Markov Model approach to variation among sites in rate of evolution. Molecular Biology and Evolution, 13(1), 93-104.

524 Forster, M., \& Sober, E. (1994). How to Tell When Simpler, More Unified, or Less Ad Hoc Theories will Provide More Accurate Predictions. The British Journal for the Philosophy of Science, 45(1), 1-35. doi:10.1093/bjps/45.1.1

Galtier, N. (2001). Maximum-Likelihood Phylogenetic Analysis Under a Covarion-like Model. Molecular Biology and Evolution, 18(5), 866-873.

Grundler, M. C., \& Rabosky, D. L. (2020). Macroevolutionary analysis of discrete traits with rate heterogeneity. BioRxiv, 2020.01.07.897777. doi:10.1101/2020.01.07.897777

Losos, J. B. (2011). Seeing the Forest for the Trees: The Limitations of Phylogenies in Comparative Biology: (American Society of Naturalists Address). The American Naturalist, 177(6), 709-727. doi:10.1086/660020

Maddison, W. P., \& FitzJohn, R. G. (2015). The Unsolved Challenge to Phylogenetic Correlation Tests for Categorical Characters. Systematic Biology, 64(1), 127-136.

544 Mahler, D. L., Revell, L. J., Glor, R. E., \& Losos, J. B. (2010). Ecological opportunity and the doi:10.1093/sysbio/syu070

Maddison, W. P., Midford, P. E., Otto, S. P., \& Oakley, T. (2007). Estimating a Binary rate of morphological evolution in the diversification of Greater Antillean anoles. Evolution: International Journal of Organic Evolution, 64(9), 2731-2745. 
24 Boyko and Beaulieu

547 Marazzi, B., Ané, C., Simon, M. F., Delgado $\square$ Salinas, A., Luckow, M., \& Sanderson, M. J.

548 (2012). Locating Evolutionary Precursors on a Phylogenetic Tree. Evolution, 66(12), 3918-3930. doi:10.1111/j.1558-5646.2012.01720.x

550 Mayr, E. (1997). This is biology: The science of the living world. Universities Press.

551 Mossel, E. (2003). On the Impossibility of Reconstructing Ancestral Data and Phylogenies. Journal of Computational Biology, 10(5), 669-676. doi:10.1089/106652703322539015

553 Mossel, E., \& Peres, Y. (2003). Information flow on trees. The Annals of Applied Probability, 13(3), 817-844. doi:10.1214/aoap/1060202828

O’Meara, B. C. (2012). Evolutionary Inferences from Phylogenies: A Review of Methods. Annual Review of Ecology, Evolution, and Systematics, 43(1), 267-285. doi:10.1146/annurev-ecolsys-110411-160331

O’Meara, B. C., Smith, S. D., Armbruster, W. S., Harder, L. D., Hardy, C. R., Hileman, L. C., ... Diggle, P. K. (2016). Non-equilibrium dynamics and floral trait interactions shape extant angiosperm diversity. Proceedings of the Royal Society B: Biological Sciences,

Penny, D., McComish, B. J., Charleston, M. A., \& Hendy, M. D. (2001). Mathematical Elegance with Biochemical Realism: The Covarion Model of Molecular Evolution. Journal of

568 Platt, J. R. (1964). Strong Inference. Science, 146(3642), 347-353.

569 Rabosky, D. L., \& Goldberg, E. E. (2015). Model Inadequacy and Mistaken Inferences of TraitDependent Speciation. Systematic Biology, 64(2), 340-355. doi:10.1093/sysbio/syu131

571 Román-Palacios, C., Scholl, J. P., \& Wiens, J. J. (2019). Evolution of diet across the animal tree of life. Evolution Letters, 3(4), 339-347. doi:10.1002/ev13.127 
25 Hidden Markov Models for phylogenetic comparative datasets

573 Sanderson, M. J., \& Donoghue, M. J. (1989). Patterns of Variation in Levels of Homoplasy.

$574 \quad$ Evolution, 43(8), 1781-1795. doi:10.1111/j.1558-5646.1989.tb02626.x

575 Sauquet, H., von Balthazar, M., Doyle, J. A., Endress, P. K., Magallón, S., Staedler, Y., \& Schönenberger, J. (2018). Challenges and questions in reconstructing the ancestral flower of angiosperms: A reply to Sokoloff et al. American Journal of Botany, 105(2), 127-135. doi:10.1002/ajb2.1023

Sauquet, H., von Balthazar, M., Magallón, S., Doyle, J. A., Endress, P. K., Bailes, E. J., ...

Schultz, T. R., Cocroft, R. B., \& Churchill, G. A. (1996). The Reconstruction of Ancestral Character States. Evolution, 50(2), 504-511. doi:10.1111/j.1558-5646.1996.tb03863.x

Siepel, A., \& Haussler, D. (2005). Phylogenetic Hidden Markov Models. In Statistical Methods in Molecular Evolution (pp. 325-351). New York: Springer-Verlag. doi:10.1007/0-38727733-1_12

Sober, E., \& Steel, M. (2011). Entropy increase and information loss in Markov models of

Sokoloff, D. D., Remizowa, M. V., Bateman, R. M., \& Rudall, P. J. (2018). Was the ancestral angiosperm flower whorled throughout? American Journal of Botany, 105(1), 5-15.

594 Steel, M., \& Penny, D. (2005). Maximum parsimony and the phylogenetic information in multistate characters. New York: Oxford University Press. 
26 Boyko and Beaulieu

599 Townsend, J. P., \& Naylor, G. (2007). Profiling Phylogenetic Informativeness. Systematic

$$
\text { Biology, 56(2), 222-231. doi:10.1080/10635150701311362 }
$$

601 Uyeda, J. C., Zenil-Ferguson, R., \& Pennell, M. W. (2018). Rethinking phylogenetic comparative methods. Systematic Biology, 67(6), 1091-1109. doi:10.1093/sysbio/syy031

603 Yang Lou, X. (2017). Hidden Markov Model Approaches for Biological Studies. Biometrics \& Biostatistics International Journal, 5(4). doi:10.15406/bbij.2017.05.00139

Zucchini, W., MacDonald, I. L., \& Langrock, R. (2017). Hidden Markov models for time series: an introduction using $R$. Chapman and Hall/CRC.

Table 1 - Model rankings from the maximum-likelihood analysis of the ancestral angiosperm

610 flower for dataset two (polymorphic species included and unknown states excluded). Models 611 separated by a "/" indicate a hidden rates model and the split distinguishes between the two state-

612 dependent process (for example, $E R / A R D$, represents a hidden rate model where $\mathrm{R} 1$ is an equal 613 rates model and R2 is an all rates differ model). Dual describe whether the model allowed for 614 multi-state transitions (for example, if dual transitions were TRUE, then changing from entirely 615 whorled phyllotaxy to entirely spiral phyllotaxy is allowed). AICc is the sample size corrected 616 Aikaike Information Criterion. AICcWt is the relative likelihood of each model and is used in 617 model averaging. Mean rate is the average transition rate for a particular model. ASR is the most 618 likely ancestral state reconstruction for a particular model and its marginal probability. $k$ rates is 619 the number of independent rate parameters being estimated for a given model.

\begin{tabular}{|c|c|c|c|c|c|c|}
\hline Model & $\begin{array}{c}\text { Dual } \\
\text { Transitions }\end{array}$ & AICc & AICcWt & Mean Rate & ASR & $\begin{array}{c}k \\
\text { rates }\end{array}$ \\
\hline ER & FALSE & 208.44 & $<0.01$ & $<0.01$ & $(1, \mathrm{R} 1) 93 \%$ & 1 \\
\hline SYM & FALSE & 138.25 & $<0.01$ & 16.67 & $(1, \mathrm{R} 1) 99 \%$ & 12 \\
\hline ARD & FALSE & 125.53 & $<0.01$ & 13.56 & $(5, \mathrm{R} 1) 100 \%$ & 24 \\
\hline ER/ER & FALSE & 118.06 & 0.01 & 37.52 & $(1, \mathrm{R} 2) 63 \%$ & 4 \\
\hline
\end{tabular}



available under aCC-BY-NC-ND 4.0 International license.

27 Hidden Markov Models for phylogenetic comparative datasets

\begin{tabular}{|c|c|c|c|c|c|c|}
\hline SYM/SYM & FALSE & 147.3 & $<0.01$ & 6.25 & $(1, \mathrm{R} 2) 93 \%$ & 26 \\
\hline ER/ARD & FALSE & 132.6 & $<0.01$ & 4.72 & $(8, \mathrm{R} 2) 87 \%$ & 27 \\
\hline ARD/ARD & FALSE & 191.76 & $<0.01$ & 13.58 & $(4, \mathrm{R} 1) 82 \%$ & 50 \\
\hline ER & TRUE & 115.2 & 0.06 & $<0.01$ & $(1, \mathrm{R} 1) 97 \%$ & 1 \\
\hline SYM & TRUE & 193.77 & $<0.01$ & 5.51 & $(5, \mathrm{R} 1) 100 \%$ & 50 \\
\hline ARD & TRUE & 212.59 & $<0.01$ & 16.36 & $(5, \mathrm{R} 1) 100 \%$ & 56 \\
\hline ER/ER & TRUE & $\mathbf{1 0 9 . 7 2}$ & $\mathbf{0 . 9 3}$ & $<\mathbf{0 . 0 1}$ & $\mathbf{( 1 , R 2 ) ~ 9 1 \%}$ & $\mathbf{4}$ \\
\hline SYM/SYM & TRUE & 383.59 & $<0.01$ & 5.47 & $(8, \mathrm{R} 1) 92 \%$ & 102 \\
\hline ER/ARD & TRUE & 220.93 & $<0.01$ & 3.13 & $(8, \mathrm{R} 2) 93 \%$ & 59 \\
\hline ARD/ARD & TRUE & 443.07 & $<0.01$ & 10.76 & $(4, \mathrm{R} 1) 86 \%$ & 114 \\
\hline
\end{tabular}

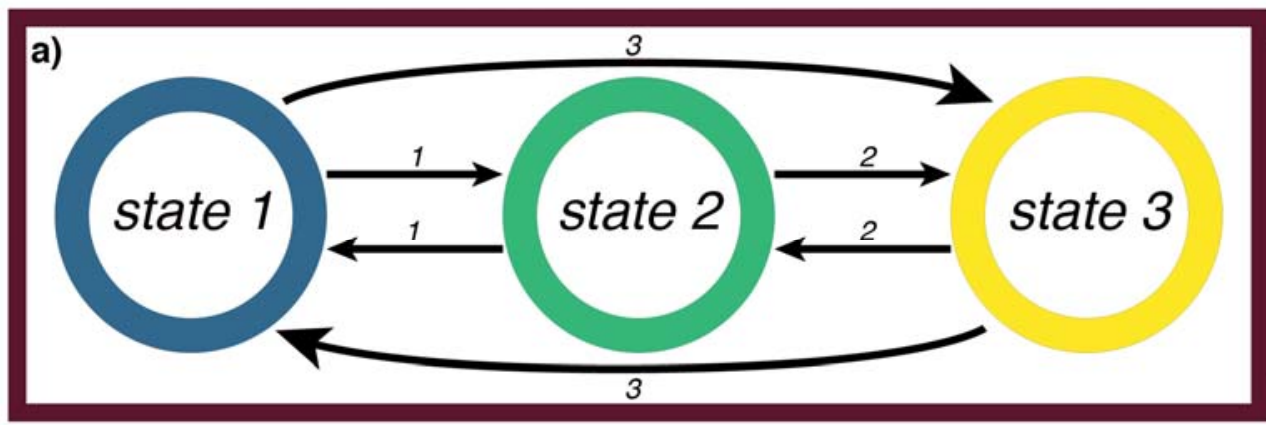

d)

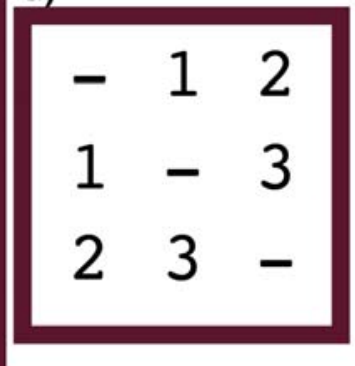

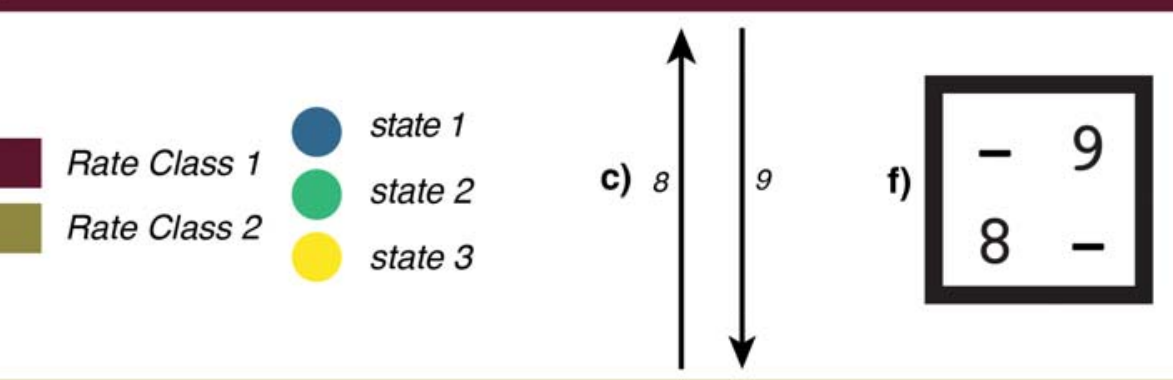

b)

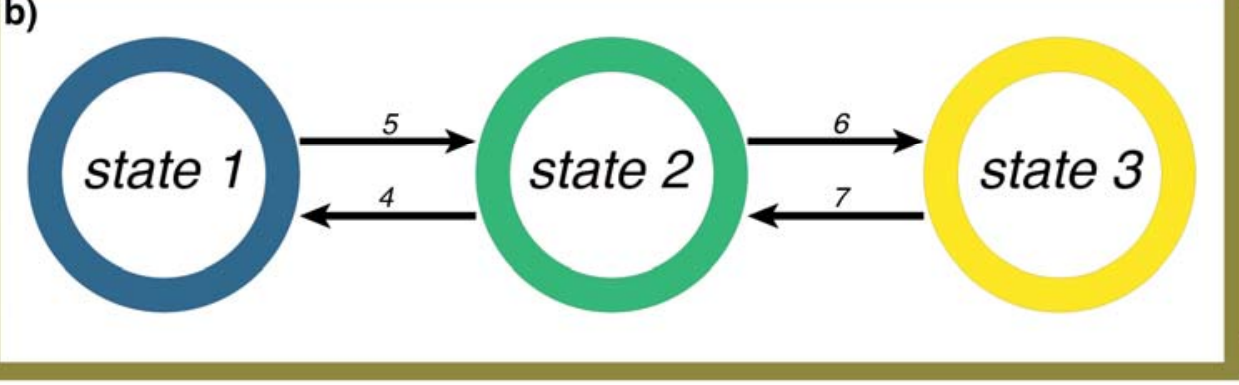

e)

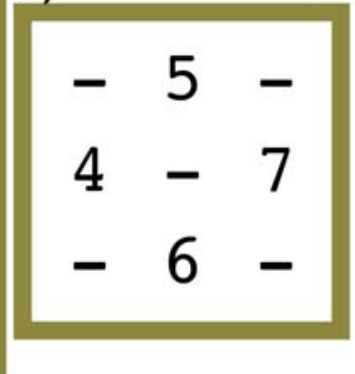

623 Figure 1. A decomposed HMM containing 3 observed states and 2 hidden rate classes. R1 is one

624 state-dependent process that describes transitions to and from observed states as being equal (a, 
28 Boyko and Beaulieu

d), whereas $\mathrm{R} 2$ is a state-dependent process that describes state 2 as a necessary intermediate (b,

627 R2 (c, f).

a)

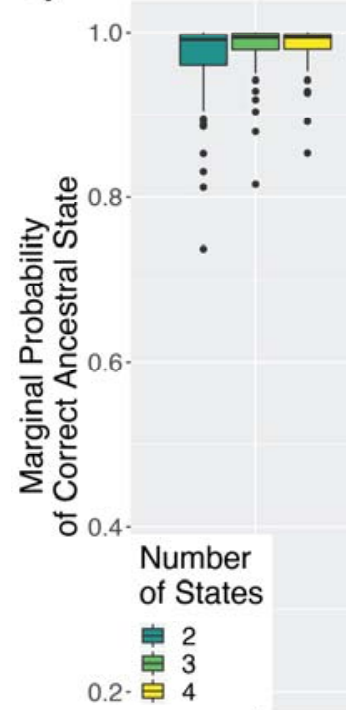

0.1

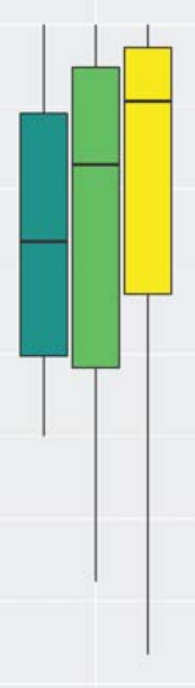

Mean Rate b)

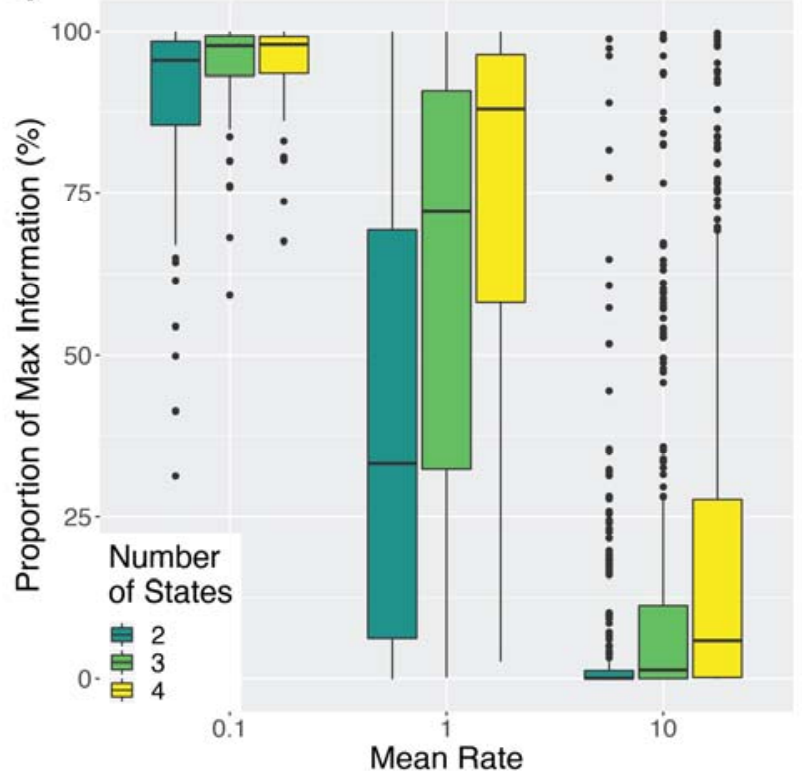

630 Figure 2. Performance of standard and hidden Markov models depending on the number of

631 states in the dataset and mean rate. Each dataset was simulated under a mean rate of $0.1,1$, or 10

632 transitions $\mathrm{Myr}^{-1}$, with 2, 3, or 4 observed states and no hidden states. a) The marginal

633 probability of estimating the correct ancestral state. b) The proportion of information gained

634 about ancestral states from each dataset and model. 
29 Hidden Markov Models for phylogenetic comparative datasets
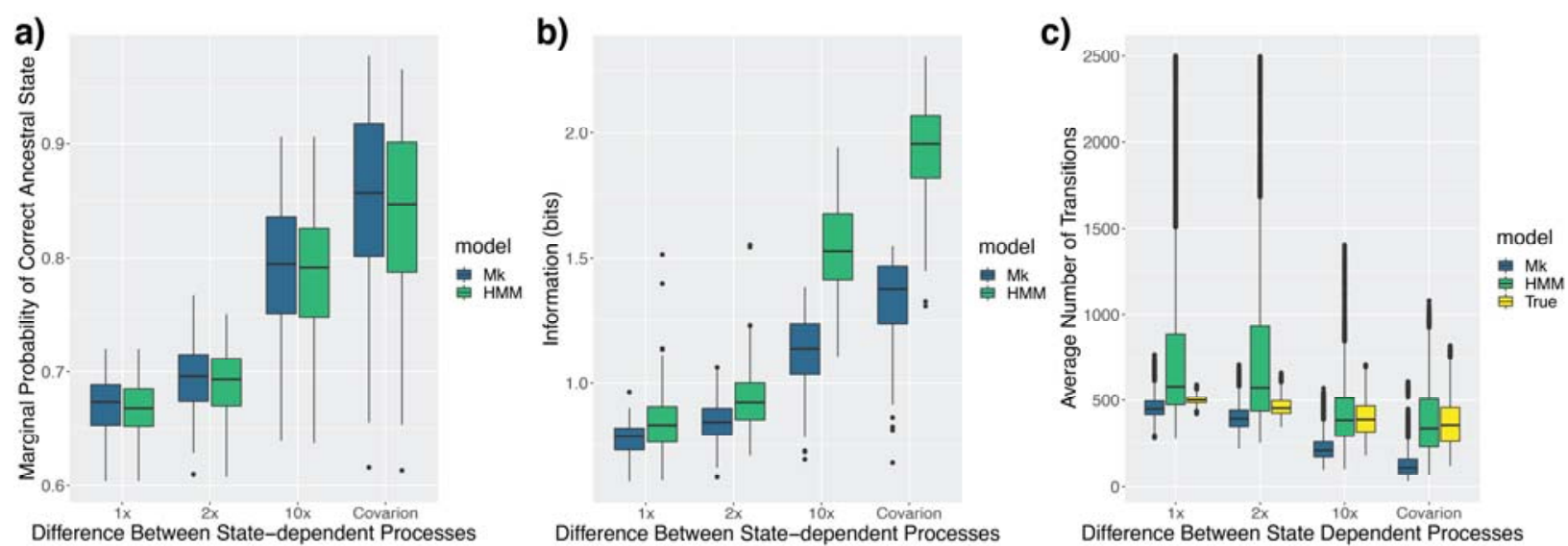

642 Figure 3. Comparison of fits from a Markov and HMM model when an HMM is the generating

643 model. We vary the difference between state-dependent processes from no difference (1x) to

644 complete asymmetry where state transitions occur in one state-dependent process only (i.e.,

645 "covarion" model; see Performance in simulation). a) The marginal probability of the correct

646 ancestral state. b) The average amount of information (bits) for ancestral states from each dataset

647 and model. c) The number of transitions averaged over 150 simmaps. 
30 Boyko and Beaulieu

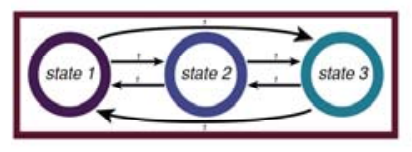

Rate Class $1=\quad$ Rate Class 2
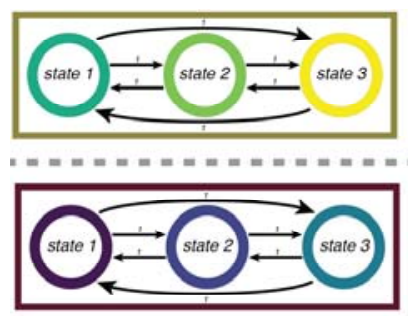

Rate Class $1=/=\square$ Rate Class 2

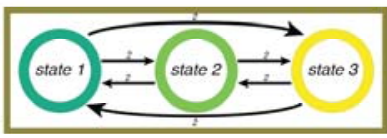

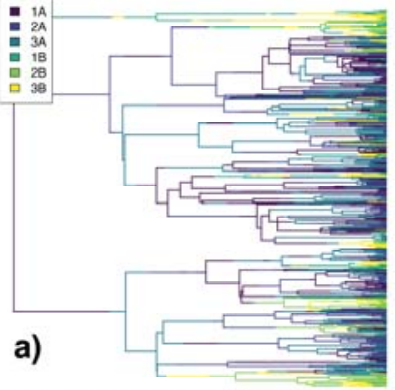
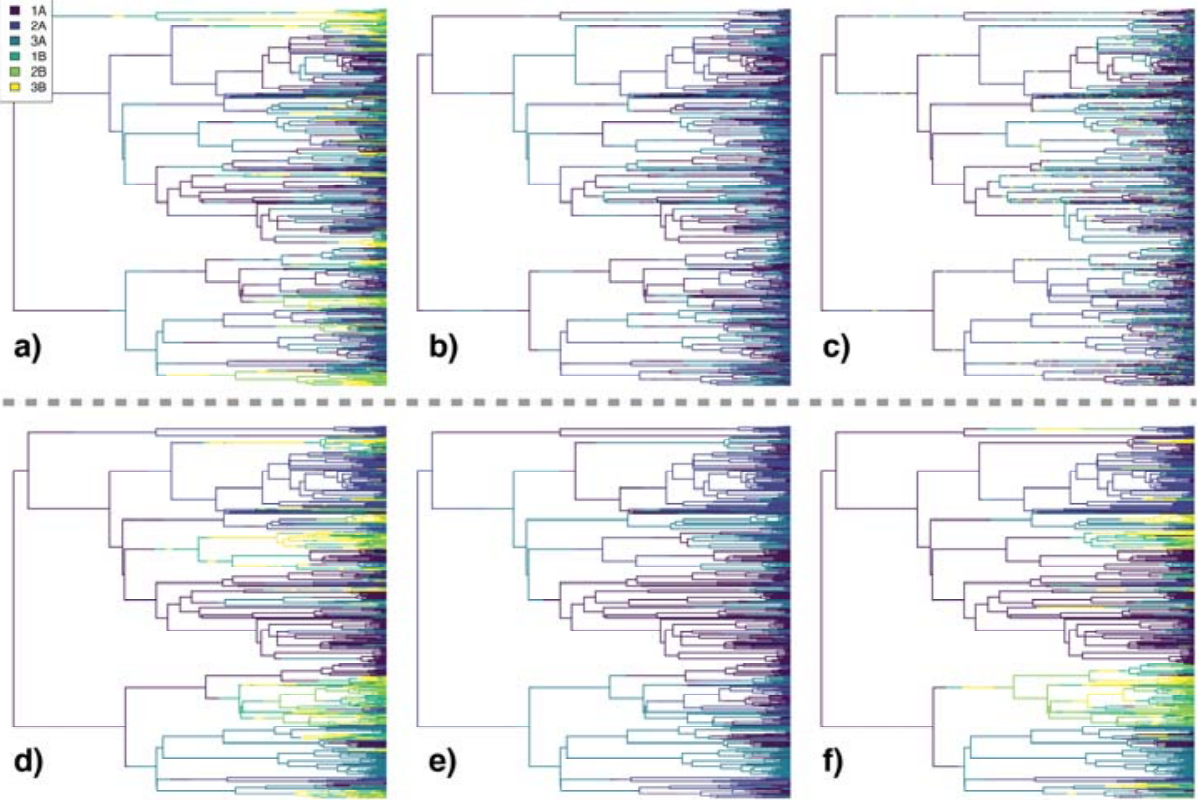

654 Figure 4. Stochastic maps demonstrating the effect of differences in the magnitude of two state-

655 dependent processes. The first row shows data is simulated where there were no differences in

656 the state-dependent processes, where (a) is the true generating model, (b) is one example of the

657 character history simulated under the MLE from standard Markov model, and (c) is one example

658 of the character history simulated under the MLE of an HMM. The second row is the same, but

659 with data simulated with a 10-fold difference between the state-dependent processes. A Markov

660 model does not contain a distinction between the hidden classes A and B, thus it is displayed

661 only in terms of the states 1A, 2A, and 3A. Comparing the HMM in (c) and (f) demonstrates that

662 an HMM will only detect a hidden state when it influences the observed, state-dependent,

663 process.

664

665 
31 Hidden Markov Models for phylogenetic comparative datasets

model

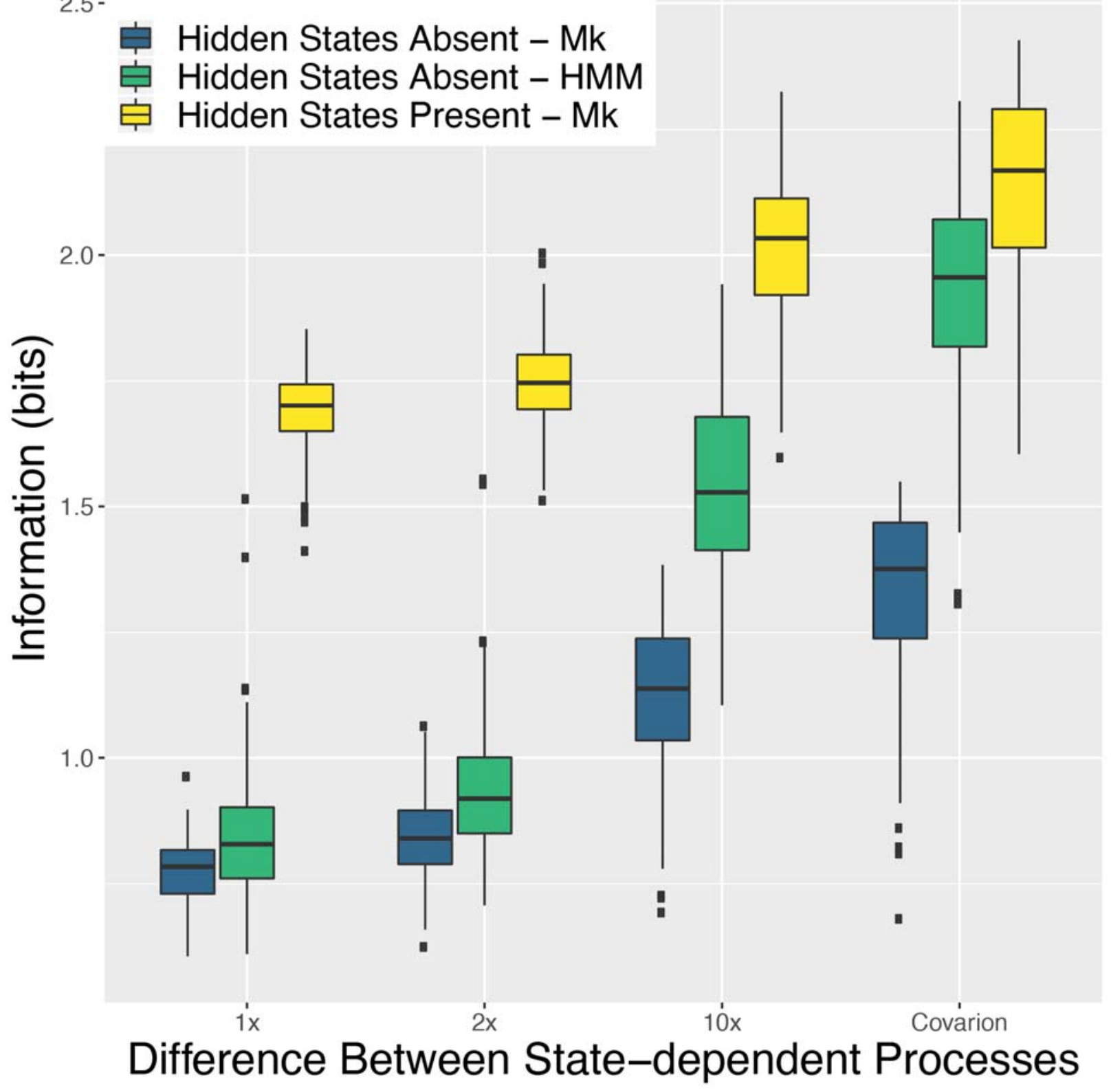

Figure 5. Average information when a hidden state is either directly observable or unobserved.

668 If the hidden state is unobserved (Hidden States Absent), we compare the information gained

669 when fitting a Markov model (Mk) or a hidden Markov model (HMM) to a dataset that was

670 generated with a hidden state, but that hidden state was removed from the dataset. When the

671 hidden state is directly observable (Hidden States Present) we fit a standard Mk to the full dataset 
bioRxiv preprint doi: https://doi.org/10.1101/2020.07.18.209874; this version posted September 28, 2020. The copyright holder for this preprint (which was not certified by peer review) is the author/funder, who has granted bioRxiv a license to display the preprint in perpetuity. It is made available under aCC-BY-NC-ND 4.0 International license.

\section{Boyko and Beaulieu}

672 that includes the potential hidden state. When the hidden state is directly observed, the datasets

673 are comprised of 6 discrete states.

Perianth \& Androecium \& Gynoecium - (0) whorled \& $(0)$ whorled \& $(0)$ whorled

- (0) whorled \& (0) whorled \& (1) spiral

- (0) whorled \& (1) spiral \& (0) whorled

- (0) whorled \& (1) spiral \& (1) spiral

- (1) spiral \& (0) whorled \& (0) whorled

(1) spiral \& (0) whorled \& (1) spiral

- (1) spiral \& (1) spiral \& (0) whorled

(1) spiral \& (1) spiral \& (1) spiral 
bioRxiv preprint doi: https://doi.org/10.1101/2020.07.18.209874; this version posted September 28, 2020. The copyright holder for this preprint (which was not certified by peer review) is the author/funder, who has granted bioRxiv a license to display the preprint in perpetuity. It is made available under aCC-BY-NC-ND 4.0 International license.

\section{Hidden Markov Models for phylogenetic comparative datasets}

676 Figure 6. Model averaged ancestral state reconstructions of dataset two (polymorphic species are

677 included, but species with unknown states are excluded). The marginal probability that the root

678 state is entirely whorled is $91.7 \%$. 